\title{
Review of: "Upper extremity deep vein thrombosis in COVID-19: Incidence and correlated risk factors in a cohort of non-ICU patients"
}

\author{
Stavroula Kastora
}

Potential competing interests: The author(s) declared that no potential competing interests exist.

Mumoli et al., 2022 has provided the first rigorous evidence highlighting the putative association between UEDVT occurrence and CPAP use. The primary outcome of this study was to identify the incidence of ultrasound-based diagnosis of UEDVT including symptomatic but also asymptomatic events. Asymptomatic UEDVT events consisted of one third [N:9] of the total UEDVT events [N: 28/257]. The level of clinical relevance and importance of identifying UEDVTs in the asymptomatic cohort may have required further expansion in the discussion.

As supported by a strong body of literature evidence, COVID-19 infection is an independent derangement driver of the physiological Virchow's triad balance. Whilst it may be postulated that CPAP in this context, may drive increased ${ }^{[1]}$ and consequently derangement of the triad, variables that have been repeatedly shown to correlate with increased UEDVT risk have not been considered in the univariate and multivariate analysis models in the presently reviewed work. These include gender, presence of cervical rib, COVID-19 severity (moderate vs. severe), days of ventilation, congenital thrombophilia. Conversely, secondary UEDVT remains a significant complication especially in patients requiring PICC lines, with an incidence similar, if not greater than centrally inserted catheters, ranging from 3 to $58 \% .{ }^{[2]}$ In the present cohort, only 8 UEDVT (28.6\%) were catheter-related, the presence of which was not associated with the onset of UEDVT as per the authors conclusions, a statement that would have benefited from further elaboration. Lastly, despite its negative predictive value, D-Dimer is rarely informative in the context of UEDVT diagnosis especially in an overt inflammatory state such as COVID-19 infection and thus not recommended as per NICE guidelines.

Overall, the "Upper extremity deep vein thrombosis in COVID-19: Incidence and correlated risk factors in a cohort of non-ICU patients." by Mumoli et al., 2022, reflects a well-written manuscript highlighting the possibility of CPAP implementation and UEDVT risk, truly raising questions about the right venous thromboembolic prophylaxis in this patient group. Nonetheless, obscurities as posed above, may be answered in future studies, and consequently improve patient management, care and safety. 


\section{References}

1. ^J E Fewell, D R Abendschein, CJ Carlson, J F Murray, et al. (1980). Continuous positive-pressure ventilation decreases right and left ventricular end-diastolic volumes in the dog.. Circ Res, vol. 46 (1), 125-132. doi:10.1161/01.res.46.1.125.

2. `Vineet Chopra, Sarah Anand, Andy Hickner, Michael Buist, et al. (2013). Risk of venous thromboembolism associated with peripherally inserted central catheters: a systematic review and meta-analysis. The Lancet, vol. 382 (9889), 311-325. doi:10.1016/s0140-6736(13)60592-9. 